\title{
The planet search program at the ESO Coudé Echelle spectrometer $^{\star}$
}

\section{The $\alpha$ Centauri system: Limits for planetary companions}

\author{
M. Endl ${ }^{1}$, M. Kürster ${ }^{2}$, S. Els ${ }^{3,4}$, A. P. Hatzes ${ }^{3}$, and W. D. Cochran ${ }^{5}$ \\ 1 Universität Wien, Institut für Astronomie, Türkenschanzstr. 17, 1180 Wien, Austria \\ 2 European Southern Observatory, Casilla 19001, Vitacura, Santiago 19, Chile \\ 3 Thüringer Landessternwarte Tautenburg, Sternwarte 5, 07778 Tautenburg, Germany \\ 4 Universität Heidelberg, Institut für Theoretische Astrophysik, Tiergartenstr. 15, 69121 Heidelberg, Germany \\ 5 McDonald Observatory, The University of Texas at Austin, Austin, TX 78712-1083, USA
}

Received 11 April 2001 / Accepted 19 May 2001

\begin{abstract}
In this article we present the results of the planet search program carried out at the ESO Coudé Echelle Spectrometer (CES) for components A and B of the $\alpha$ Centauri system. Since November 1992 we have obtained high precision differential radial velocities $(R V \mathrm{~s})$ for both stars. We demonstrate that, after subtraction of the binary orbital motion, no additional residual $R V$ signal is present within our detection limits, which could have been attributed to giant planets orbiting either of the two stars. We performed numerical simulations to determine the detection threshold of the CES survey for planets around both stars. In combination with known dynamical limitations for stable planetary orbits (Wiegert \& Holman 1997) we can now set strong constraints on the existence of Jupiter-mass-type planets in the $\alpha$ Centauri system.
\end{abstract}

Key words. stars: planetary systems - stars: individual: $\alpha$ Centauri - stars: binaries: close - techniques: radial velocities

\section{Introduction}

The $\alpha$ Centauri system is our closest neighbour in space and constitutes an interesting target for the search for extrasolar giant planets for several reasons: a discovered planet would be a prime target for a follow-up imaging/spectroscopy program due to the proximity of $\alpha$ Centauri $(1.347 \mathrm{pc})$, its binary nature could lead to new insights into planetary formation processes in binary systems and the dynamical stability of planetary orbits in such an environment could be examined empirically. Although planetary orbits at separations similar to our Jupiter $(\approx 5 \mathrm{AU})$ are dynamically unstable in the $\alpha$ Centauri AB binary system (e.g. Wiegert \& Holman 1997), the discoveries of giant planets orbiting at very small separations (e.g. Mayor \& Queloz 1995) leaves the possibility open that Jupiter-mass companions exist at smaller orbital radii around either $\alpha$ Centauri A or B.

The planet search program at the Coudé Echelle Spectrometer (CES) at ESO La Silla is a precise Doppler search for variations in the differential radial velocities of

Send offprint requests to: M. Endl,

e-mail: endl@astro.univie.ac.at

* Based on observations collected at the European Southern Observatory, La Silla.
40 stars in the southern hemisphere (Kürster et al. 1994; Hatzes et al. 1996; Endl et al. 2000). In this sample we have discovered one extrasolar giant planet orbiting the young (ZAMS) and active G0V star $\iota$ Horologii in an Earth-like orbit (Kürster et al. 2000) and the $R V$ results we obtained for $\epsilon$ Eridani contributed to the evidence for a long period $(P \approx 6.9 \mathrm{yrs})$ planet in an eccentric $(e=0.6)$ orbit around this star (Hatzes et al. 2000). The $\alpha$ Centauri system has been monitored since the beginning of the program in November 1992. Observations were performed by using the $1.4 \mathrm{~m}$ Coudé Auxiliary Telescope (CAT) and the CES equipped with the Long Camera yielding a resolving power of $R=100000$. The CES instrument was upgraded in April 1998 by installing the new Very Long Camera yielding a higher resolving power $(R=230000)$. Our results presented in this article all refer to the CES configuration (with the Long Camera, $R=100000$ ) prior to this modification. In Kürster et al. (1999) we have already presented the results for Proxima Centauri, the possible third member in this system. A full analysis of the complete CES planet search data will be presented in a forthcoming paper (Endl et al., in prep.).

The plan for this article is the following: first we present the radial velocity results for $\alpha$ Centauri A \& B and discuss the existence of residual $R V$ signals which 
would indicate Jupitermass-type planets. We continue with numerical simulations to determine the detectability of such planets in the $\alpha$ Centauri system by the CES program. Finally we combine our results with the study of Wiegert \& Holman (1997) who presented dynamical limitations for stable planetary orbits around both components. This combination of observational data and dynamical simulations results in strong constraints for the presence of giant planets in the $\alpha$ Centauri binary system.

\section{Radial velocities and residual signals}

The Coudé Echelle Spectrometer (CES) at the ESO La Silla observatory is a conventional non-stabilized spectrograph and in order to achieve the necessary $R V$ precision we use a temperature controlled and pressure stabilized iodine $\left(I_{2}\right)$ vapor absorption cell to self-calibrate the instrument. A detailed description of the instrument setup, observational process, and the analysis technique we use to extract the $R V$ information from CES planet search data can be found in Paper I of this series (Endl et al. 2000).

The inner binary (semimajor axis $=23 \mathrm{AU}$ ) of the $\alpha$ Centauri system consists of a G2V primary (HR 5459, HD 128620, $V=-0.01$ ) and a K1V secondary (HR 5460, HD 128621, $V=1.33)$ moving in an eccentric $(e=0.518)$ orbit with a period of almost 80 years (Heintz 1982; Pourbaix et al. 1999). Their masses have been determined by Pourbaix et al. (1999) to be $1.16 M_{\odot}(\mathrm{A})$ and $0.97 M_{\odot}(\mathrm{B})$. These values probably represent upper limits as stated by Guenther \& Demarque (2000). However, we adopt these higher mass values for this work because slightly lower stellar masses would only reduce the planetary mass limits we derive in Sect. 3 to a minor extent and the aim is to set quantitative upper limits for companion masses. The third possible member of the system, Proxima Centauri (M5V, $V=11.05)$, is located at a much larger distance of $\approx 12000$ AU. It has been proposed that Proxima Centauri forms a stellar moving group with the inner binary (Anosova et al. 1994), rather than being gravitationally bound in a triple system. CES $R V$ results for Proxima Centauri and companion limits based upon this data were already presented in Kürster et al. (1999), hence we will not consider this star in this work.

Over the time of $51 / 2$ years (Nov. 1992-April 1998) we obtained a total of 205 individual $R V$ measurements for $\alpha$ Centauri A and 291 for $\alpha$ Centauri B taken during 48 and 43 nights respectively. Due to their brightness and hence short exposure times, we took several spectra each night. For the following analysis we average the results of one night by taking the mean $R V$ value of that night while the error is computed by propagating the errors of each individual measurement, leaving us with $48 R V$ measurements for component A and 43 for component B. Table 1 lists the times of observations and the averaged $R V$ results with their measurement errors. Note that these are differential measurements and that an arbitrary velocity zero-point was subtracted from the $R V$ results for each
Table 1. Differential $R V$ measurements of $\alpha$ Centauri A \& B.

\begin{tabular}{|c|c|c|}
\hline BJD & $\alpha \mathrm{CEN} \mathrm{A:} \Delta R V\left[\mathrm{~m} \mathrm{~s}^{-1}\right]$ & $\alpha$ CEN B: $\Delta R V\left[\mathrm{~m} \mathrm{~s}^{-1}\right]$ \\
\hline 2449054.8 & $-328.5 \pm 10.6$ & $390.0 \pm 8.9$ \\
\hline 2449055.7 & $-329.3 \pm 12.2$ & $389.9 \pm 8.2$ \\
\hline 2449109.8 & $-310.2 \pm 7.4$ & $372.4 \pm 10.6$ \\
\hline $\begin{array}{lll}2 & 449 & 179.7\end{array}$ & $-312.8 \pm 22.4$ & $341.9 \pm 11.0$ \\
\hline 2449246.5 & $-292.2 \pm 9.6$ & $323.6 \pm 7.9$ \\
\hline 2449358.9 & & $277.1 \pm 9.1$ \\
\hline 2449412.8 & $-230.4 \pm 10.9$ & $261.6 \pm 9.6$ \\
\hline 2449492.6 & $-223.7 \pm 11.1$ & $237.0 \pm 8.7$ \\
\hline 2449493.5 & $-225.0 \pm 12.3$ & \\
\hline 2449548.6 & & $224.5 \pm 8.4$ \\
\hline 2449602.5 & $-187.2 \pm 9.6$ & $214.3 \pm 11.1$ \\
\hline 2449794.9 & $-97.3 \pm 19.8$ & $150.3 \pm 11.6$ \\
\hline 2449795.8 & $-83.7 \pm 19.1$ & \\
\hline 2449855.6 & $-115.6 \pm 17.0$ & $115.4 \pm 11.7$ \\
\hline 2449906.5 & $-90.9 \pm 11.6$ & $111.8 \pm 9.0$ \\
\hline 2449972.5 & $-42.8 \pm 25.4$ & \\
\hline 2450085.9 & $-47.8 \pm 11.1$ & $40.2 \pm 8.0$ \\
\hline 2450145.9 & $-20.6 \pm 10.6$ & $21.9 \pm 8,4$ \\
\hline 2450146.8 & $-26.3 \pm 10.8$ & $19.3 \pm 10.1$ \\
\hline 2450229.7 & & $-7.4 \pm 8.5$ \\
\hline 2450230.8 & $-3.0 \pm 9.5$ & $-3.2 \pm 9.7$ \\
\hline 2450313.6 & $22.4 \pm 9.0$ & $-21.0 \pm 10.6$ \\
\hline 2450314.6 & $37.6 \pm 8.9$ & $-28.6 \pm 10.1$ \\
\hline 2450319.6 & $35.1 \pm 9.8$ & \\
\hline 2450323.6 & $25.0 \pm 9.8$ & \\
\hline 2450325.6 & $37.6 \pm 11.6$ & \\
\hline 2450330.5 & $35.0 \pm 9.9$ & \\
\hline 2450348.5 & $52.2 \pm 18.0$ & $0.2 \pm 13.8$ \\
\hline 2450349.5 & $40.0 \pm 15.8$ & $-5.8 \pm 11.4$ \\
\hline 2450357.5 & & $-53.4 \pm 7.5$ \\
\hline 2450358.5 & $27.8 \pm 9.4$ & \\
\hline 2450359.5 & $30.8 \pm 9.7$ & \\
\hline 2450477.9 & $72.5 \pm 9.6$ & $-107.8 \pm 10.4$ \\
\hline 2450478.9 & $70.4 \pm 11.3$ & $-100.1 \pm 10.0$ \\
\hline 2450523.9 & $77.2 \pm 11.4$ & $-106.8 \pm 7.3$ \\
\hline 2450524.9 & $80.0 \pm 12.3$ & $-111.2 \pm 6.9$ \\
\hline 2450550.8 & $88.9 \pm 11.2$ & $-128.5 \pm 9.4$ \\
\hline 2450551.7 & $83.3 \pm 9.7$ & \\
\hline 2450570.8 & $105.2 \pm 8.1$ & $-133.8 \pm 10.4$ \\
\hline 2450595.9 & $111.3 \pm 14.6$ & $-128.9 \pm 11.6$ \\
\hline 2450596.7 & $114.8 \pm 12.8$ & $-145.3 \pm 11.4$ \\
\hline 2450647.7 & $126.8 \pm 12.9$ & $-157.5 \pm 9.2$ \\
\hline 2450648.5 & & $-150.9 \pm 7.4$ \\
\hline 2450690.5 & $145.0 \pm 14.0$ & $-159.9 \pm 15.5$ \\
\hline 2450703.5 & $133.2 \pm 12.6$ & $-189.8 \pm 11.6$ \\
\hline 2450704.5 & $144.9 \pm 9.3$ & $-185.5 \pm 11.8$ \\
\hline 2450709.5 & $153.8 \pm 11.4$ & $-161.0 \pm 9.1$ \\
\hline 2450733.5 & $160.6 \pm 11.7$ & $-180.5 \pm 7.3$ \\
\hline 2450854.8 & $184.3 \pm 11.5$ & $-233.2 \pm 10.8$ \\
\hline 2450856.8 & $194.1 \pm 12.4$ & $-237.9 \pm 11.6$ \\
\hline 2450897.9 & $180.7 \pm 10.6$ & $-251.3 \pm 10.4$ \\
\hline 2450899.8 & $195.1 \pm 16.7$ & $-243.2 \pm 10.4$ \\
\hline 2450907.7 & $201.2 \pm 13.1$ & $-259.1 \pm 8.4$ \\
\hline
\end{tabular}

star. So the differences between the velocity values for component $\mathrm{A}$ and $\mathrm{B}$ are also arbritary, only the differential $R V$ data for each star possess meaningful information for the search for planetary companions. Preliminary CES $R V$ results for $\alpha$ Centauri, based on a different analysis technique yielding a slightly lower $R V$ precision, were already shown in Cochran \& Hatzes (1999).

Pourbaix et al. (1999) presented the most recent orbital parameters for the binary, based on the combination of several different $R V$ sets, starting as early as 1904 . We adopted their orbital values to compute the binary motion and to compare it with our $R V$ data. For this comparison we adjusted the velocity zero-point to fit our $R V$ values, by minimisation of the $\chi^{2}$-function. The results and the $R V$ residuals after subtraction of the binary orbit are shown in Fig. 1 (for $\alpha$ Centauri A) and Fig. 2 (for $\alpha$ Centauri B). For component A the residual $R V$ scatter is $11.8 \mathrm{~m} \mathrm{~s}^{-1}$ 


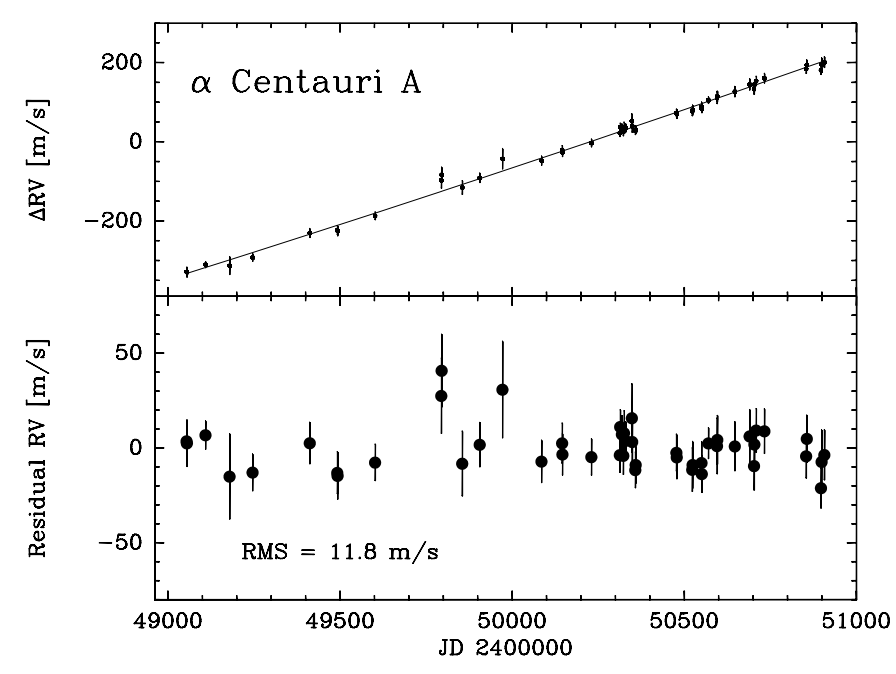

Fig. 1. Differential radial velocities of $\alpha$ Centauri A (upper panel). The solid line represents the binary orbit based on Pourbaix et al. (1999). The lower panel shows the $R V$ residuals after subtraction of this orbit. The residual scatter of $11.8 \mathrm{~m} \mathrm{~s}^{-1}$ agrees well with the mean measurement error of $12.3 \mathrm{~m} \mathrm{~s}^{-1}$.

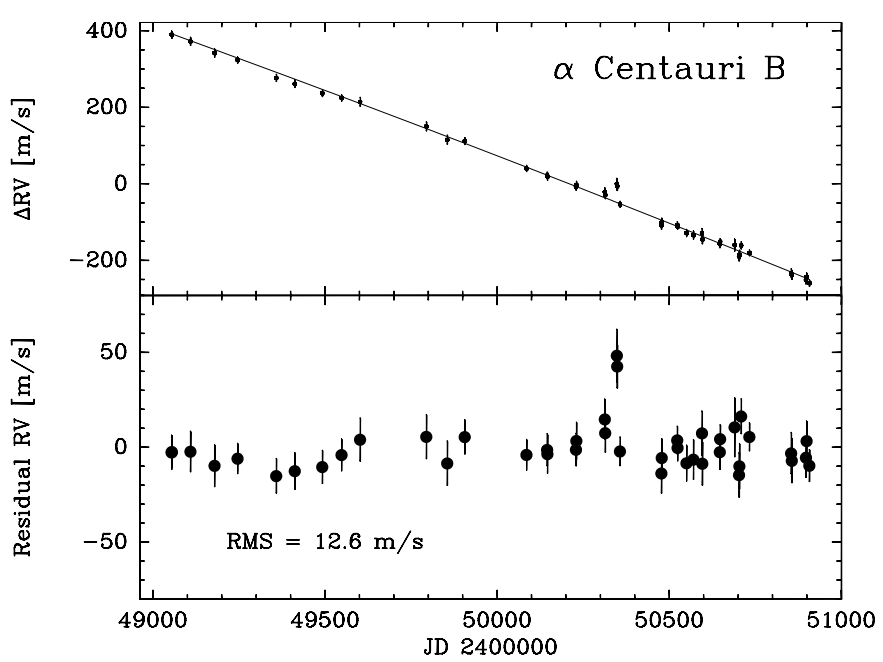

Fig. 2. Equivalent plot as Fig. 1 for $\alpha$ Centauri B. This time the residual $R V$ scatter of $12.6 \mathrm{~m} \mathrm{~s}^{-1}$ is slightly larger than the mean measurement error of $9.9 \mathrm{~m} \mathrm{~s}^{-1}$.

and for component $\mathrm{B} 12.6 \mathrm{~m} \mathrm{~s}^{-1}$. The residuals are in both cases comparable to the mean measurement errors of $12.3 \mathrm{~m} \mathrm{~s}^{-1}(\mathrm{~A})$ and $9.9 \mathrm{~m} \mathrm{~s}^{-1}(\mathrm{~B})$.

\subsection{Search for a periodic residual signal}

The similarity of the remaining $R V$ scatter around the known binary orbit with the measurement errors already indicates the absence of a strong $R V$ signal caused by an orbiting giant planet around either of both stars. We performed a period search in the range of $2-5000$ days in the $R V$ residuals by using the Lomb-Scargle periodogram (Lomb 1976; Scargle 1982). For $\alpha$ Centauri A we found the highest peak in the power spectrum to be at a period of 16.4 days and for $\alpha$ Centauri B at 17.3 days. Now we clar-

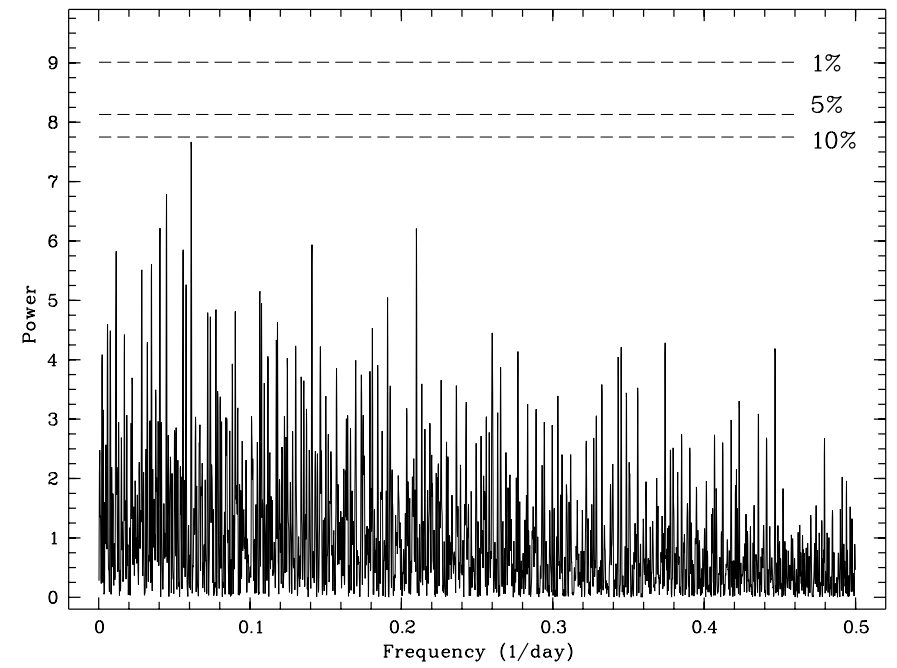

Fig. 3. Periodogram results for the $R V$ residuals of $\alpha$ Centauri A. The highest peak in the power spectrum is located at a frequency of $0.0611[1 /$ day $](P=16.4$ days $)$. The false alarm probability (FAP) of this peak is $11.37 \%$, rendering it statistically insignificant. The different FAP niveaus are displayed as horizontal dashed lines.

ify whether these signals represent "noise" peaks in the periodogram or not. We do this by estimating the "false alarm probability" (FAP) of the chance occurence of peaks with the same power as the two maximum peaks we have found. In order to establish the FAP levels of peaks in the two power spectra we employ a bootstrap randomization method. In this randomization scheme the $R V$ values are randomly redistributed while keeping the times of observations fixed (Kürster et al. 1997; Murdoch et al. 1993). For both $R V$ data sets we performed 10000 runs of this bootstrap technique and compared the power maxima of the randomized $R V$ sets with the original periodogram, thus obtaining the FAP levels. The FAP of the 16.4 days signal at $\alpha$ Centauri A was determined to be $11.38 \%$ and for the 17.3 days signal at $\alpha$ Centauri B it is $4.24 \%$. The periodogram for the $\alpha$ Centauri A data along with different FAP levels is shown in Fig. 3 and for $\alpha$ Centauri B in Fig. 4. Both signals fail to reach the FAP $=0.01$ level. Thus we cannot confirm a statistical significant residual signal in our data and we can rule out the presence of massive substellar companions. But what kind of planets exactly can we rule out now? We will deal with this question in the following section. Still, the compatibility of both periods found in the $R V$ residuals is striking. Although their FAP-level renders them as random noise peaks, is it possible that we found a real signal on a low significance level? Maybe we have detected the rotational period of the two stars or one of its harmonics? Hallam et al. (1991) obtained $P_{\text {rot }} \approx 29$ days for $\alpha$ Centauri A and Guinan \& Messina (1995) reported a $P_{\text {rot-value of }}$ $\approx 37$ days for component B based on IUE spectra. So the two signals - if they do not represent noise - are near the $1 / 2$-harmonics of the stellar rotational period. However,

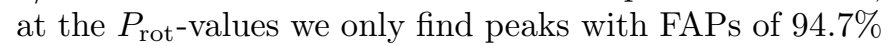
(A) and $100 \%$ (B). 


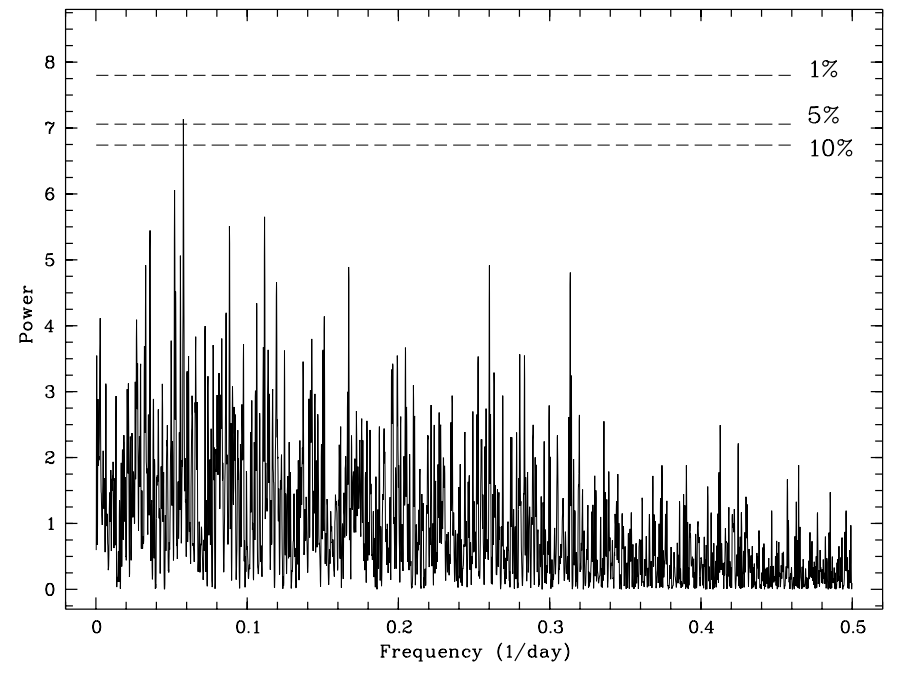

Fig. 4. Periodogram results for the $R V$ residuals of $\alpha$ Centauri B. Again the FAP values for different power levels are shown as horizontal dashed lines. The highest peak at a frequency of $0.0578[1 /$ day $](P=17.3$ days $)$ has a FAP of $4.24 \%$.

\section{Detection threshold and planetary companion limits}

To establish the CES program detection threshold for planetary companions in the $\alpha$ Centauri system we proceeded as follows (see also Endl et al. 2001): we start from the null-hypothesis that the remaining $R V$ scatter after subtraction of the binary orbit represents our measurement uncertainty. We performed numerical simulations of planetary orbits with $K$ (the $R V$ semi-amplitude), the orbital phase and $P$ (the orbital period) as model parameters (orbital eccentricity $e$ is set to 0 ). These simulated signals were added to our actual $R V$ measurements, thus using the $R V$ residuals as the noise term. This has the advantage that we don't have to assume that the error distribution follows a certain pattern, like for instance a Gaussian distribution. In fact, as can be seen in the lower panels of Figs. 1 and 2 the errors don't seem to follow a Gaussian distribution. After the synthetic $R V$ data set is created, we again subtract the binary motion and perform in the newly obtained $R V$ residuals a period search in the range of 2-5000 days, equivalent to the procedure described in the last section for the original data set. We then check whether the input signal was detected by the periodogram analysis. For each set of $K$ and $P$ parameters we create 8 simulated signals at different orbital phases, each shifted by $\pi / 4$. We define a planet as detectable by the CES program if the FAP of the peak in the power spectrum representing our input signal is less than $1 \%$. For each simulated signal we perform 1000 bootstrap randomization runs to estimate the FAP levels. If the FAP exceeds $1 \%$ at only one of the 8 trial phases we classify a planet as undetectable at these $P$ and $K$ values. By increasing the $K$ parameter until the FAP is less than $1 \%$ for all orbital phases at a given $P$ value we obtain quantitative upper limits for giant planets orbiting $\alpha$ Centauri A
\& B. We compare these results with a Monte Carlo type approach, where we used instead of our own $R V$ distribution, a Gaussian noise term (with the according sigma of $11.8 \mathrm{~m} \mathrm{~s}^{-1}$ for component $\mathrm{A}$ and $12.6 \mathrm{~m} \mathrm{~s}^{-1}$ for component B). The resulting upper limits for planetary companions using both methods are displayed in Fig. 5 for $\alpha$ Centauri A and in Fig. 6 for $\alpha$ Centauri B. A well known constraint of the $R V$ method is the unknown viewing angle $i$ to the orbital plane of the planet, thus we derive minimum mass estimates $(m \sin i)$. The difference seen between the results of the two methods employed to determine upper limits (solid and dashed lines in Figs. 5 and 6) can be explained by the differences in the underlying noise distribution. The method using our own (non-Gaussian) $R V$ data as noise term occasionally leads to the effect that outlying data points (e.g. the $2 R V$ results for $\alpha$ Centauri B at BJD 2450348 and 2450349 as seen in Fig. 2) are counteracting an $R V$ minimum (or maximum) at a certain phase angle, rendering it undetectable for the periodogram. And this can play a major role in the limit determination, because one phase is enough to reject a planet as detectable. This effect is less prominent in normally distributed errors. Since the $R V$ residuals don't follow a Gaussian distribution we conclude that the first method delivers more realistic limit results in the case of the CES program.

For $\alpha$ Centauri A we can exclude planets in circular orbits with $m \sin i>1 M_{\text {Jup }}$ at separations less than $2 \mathrm{AU}$ and within $4 \mathrm{AU}$ we rule out the presence of planets having an $m \sin i>2 M_{\text {Jup }}$. For component B the corresponding limits are: no planets with $m \sin i>1.5 M_{\text {Jup }}$ within $2 \mathrm{AU}$ and no planets with $m \sin i>2.5 M_{\text {Jup }}$ inside 4 AU. We stopped the limit analysis at $P$ values representing orbital radii of 4.5 and $4.0 \mathrm{AU}$ for components $\mathrm{A}$ and $\mathrm{B}$, respectively, due to known dynamical limitations for stable orbits around the two components (we will discuss this in more detail in the next section).

Results coming from the Mount John University Observatory (Murdoch et al. 1993) have earlier set strong constraints on the existence of brown dwarfs in the $\alpha$ Centauri system. Now the CES results also reach down into the sub-Jupitermass regime and especially we find no evidence for short period "51 Peg-type" planets with $m \sin i>0.5 M_{\text {Jup }}$ at orbital separations less than $0.4 \mathrm{AU}$.

\subsection{Combination with dynamical limitations}

The long-term stability of planetary orbits within (and around) close binary systems has always been of special interest for numerical dynamical studies (e.g. Dvorak 1986; Holman \& Wiegert 1999). In particular, the $\alpha$ Centauri system was subject of such investigations in Benest (1988) and Wiegert \& Holman (1997). A precision Doppler search like the CES program is targeting the individual stellar companions and is - of course - restricted to the detection of planets in S-type orbits (i.e. around one of the stars; after Dvorak 1986). The most recent stability study was 


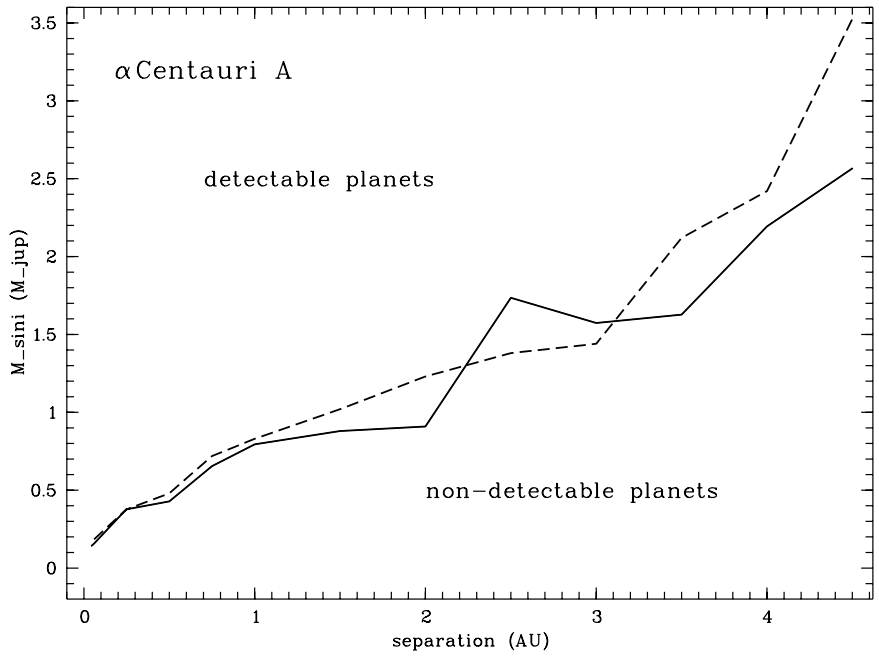

Fig. 5. Upper limits for giant planets orbiting $\alpha$ Centauri A. All synthetic planetary signals above the solid line have been detected with a significance higher than $99 \%$. The dashed curve represents the limits we derive by using a Gaussian noise term with a sigma of $11.8 \mathrm{~m} \mathrm{~s}^{-1}$ (see text for details). Note that the $y$-axis shows only the projected minimum mass of the planet $(m \sin i)$ in units of Jupiter masses. Planets orbiting in the binary plane $\left(i=79.23^{\circ}\right)$ would have masses only slightly larger than their minimum mass indicated by the solid line.

performed by Wiegert \& Holman (1997). They conducted numerical integrations over a time span of 32000 binary periods $(\approx 2.5 \mathrm{Myr})$ and determined the regions where test particles were ejected or suffered close encounters with one of the stars during the integration. Stable regions were found close to each of the stars and test particles moving in retrograde orbits survived at larger separations due to the shorter encounter times with the perturbing companion star. For planets orbiting in the binary plane (which corresponds to a viewing angle $i=79.23^{\circ}$ ) they found the stable regions to have a maximum separation of roughly $3 \mathrm{AU}$ for prograde orbits and $4 \mathrm{AU}$ for retrograde orbits. Close to each star orbits with higher inclinations $j \approx 60^{\circ}$ (with respect to the binary plane) are stable over the entire integration time. For $\alpha$ Centauri A the maximum stable orbital radii were determined for prograde orbits with inclinations of $j=0^{\circ}, 30^{\circ}, 45^{\circ}$ and $60^{\circ}$ as $a=2.9,2.5,2.1$ and 1.25 AU. For retrograde orbits the according maximum separations are: $a=4.2,3.8,2.3$ and 1.1 AU. Around component B prograde orbits were found stable with inclinations of $j=0^{\circ}, 15^{\circ}, 30^{\circ}, 45^{\circ}$ and $60^{\circ}$ outwards to $a=2.8,2.8,2.6,2.0$ and $1.2 \mathrm{AU}$. Again for retrograde orbits the allowed maximum separations for inclinations of $j=0^{\circ}, 15^{\circ}, 30^{\circ}, 45^{\circ}$ are larger: $a=4.0,4.0,3.5,3.0 \mathrm{AU}$, while higher inclined retrograde orbits with $j=60^{\circ}$ are unstable (see Figs. 1 and 3 in Wiegert \& Holman 1997 for details).

From this information about the dependence of stable regions on the orbital inclination we can now combine these limits with our $R V$ results and transform the $m \sin i$ $a$ plane of Figs. 5 and 6 into a mass-separation $(m-a)$ plane for possible planets within the $\alpha$ Centauri system. This is

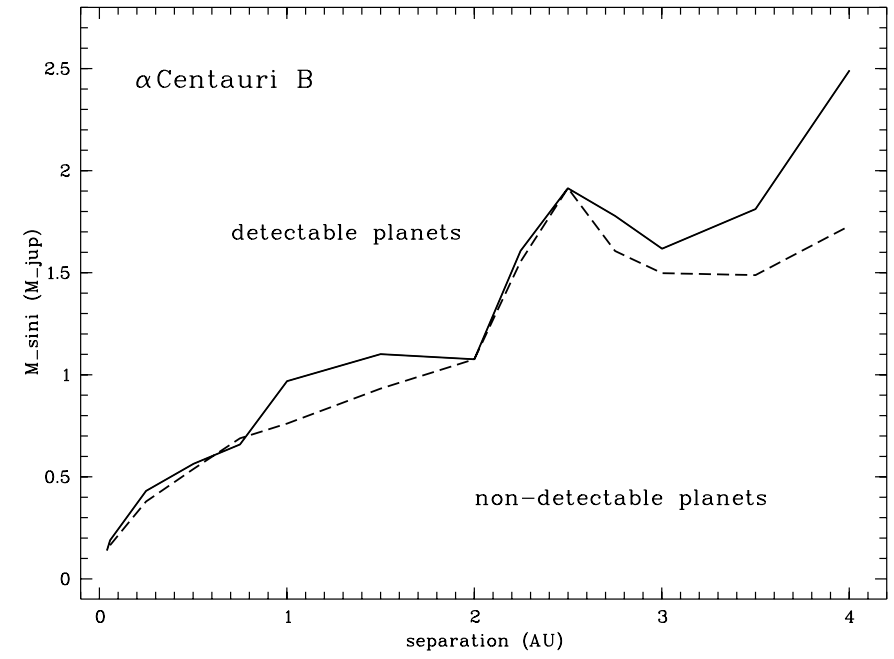

Fig. 6. Companion limits for $\alpha$ Centauri B. All planets above the solid line would have been detected by the CES program. The dashed line again shows the results taking a Gaussian noise term of $12.6 \mathrm{~m} \mathrm{~s}^{-1}$ for the simulations.

simply done by dividing the $m \sin i$-masses by the $\sin i$ values with $i=79.23^{\circ}-j$ and $j$ the maximum allowed orbital inclination at that separation. For this purpose we use the limit values based on the bootstrap method (solid lines in Figs. 5 and 6). Figures 7 and 8 display the two zones of still possible planets. This means for $\alpha$ Centauri A that no planets having masses $>2.5 M_{\text {Jup }}$ exist at any orbital radius and inclination, and for component $\mathrm{B}$ all planets with masses $>3.5 M_{\text {Jup }}$ can be excluded.

\section{Discussion}

So what kind of planets can still exist in the $\alpha$ Centauri system? Although we cannot rule out giant planets with masses smaller than $2.5 M_{\mathrm{Jup}}$ and $3.5 M_{\mathrm{Jup}}$, respectively, it is possible that the real limits are much lower than that. At least in one case, in the case of the transiting planet around HD 209458 (Charbonneau et al. 2000) it was determined that the planet orbits almost coplanar with the stellar equator and that it is moving in the same direction as the star rotates (Queloz et al. 2000; Marcy et al. 2001). Of course the same is true for our own Solar System, and if we adopt this paradigm also for the $\alpha$ Centauri system, the planetary mass limits decrease dramatically, especially at smaller orbital radii. There is evidence that the equatorial and the orbital planes are coplanar in solar-type binary systems with separations less than $\approx 30$ AU (Hale 1994). The viewing angle to the binary plane of $\alpha$ Centauri is well determined with $79.23^{\circ}$. If we assume now that possible planets would orbit in the same plane, then the $m \sin i$ values of Figs. 5 and 6 divided by $\sin \left(79.23^{\circ}\right)(=0.982)$ would yield the slightly larger "real" mass value. This roughly transforms the limits displayed in Figs. 5 and 6 into real mass limits without the uncertainty of the $\sin i$-effect. This would consequently even exclude all "51 Peg-type" planets with masses larger than $\approx 0.6 M_{\text {Saturn }}$ at separations less than $0.06 \mathrm{AU}$. 


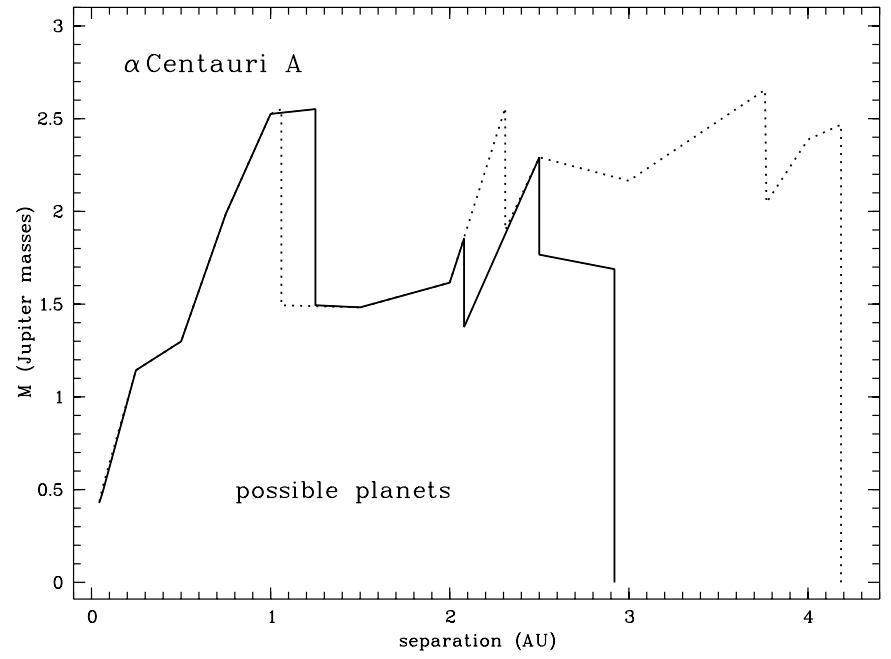

Fig. 7. Mass-separation plane of the remaining possible planets around $\alpha$ Centauri A. This plane is the result of the combination of the $m \sin i$-curve (solid line) of Fig. 5 with the stability zones of Wiegert \& Holman (1997). We can exclude the existence of all planets above and to the right of the solid line (dotted line) for prograde (retrograde) orbits.

Marzari \& Scholl (2000) have demonstrated that planetesimal accretion is still possible within the stable regions of the $\alpha$ Centauri binary. They showed that gas drag inside the protoplanetary disk counteracts the strong perturbations caused by the secondary. Hence, despite the fact that we did not detect any giant planets residing inside the binary, the $\alpha$ Centauri system can still be hosting lower mass giants and of course terrestrial planets.

Finally, planets orbiting the center of mass of the binary (P-type orbits after Dvorak 1986) at much larger separations $(a>70 \mathrm{AU})$ cannot be detected by a Doppler search like the CES program and thus remain another possibility.

The planetary limits presented in this work are based on more than 5 years of data collected with the $1.4 \mathrm{~m} \mathrm{CAT}$ telescope and the CES in Long Camera configuration. The CES (with the new Very Long Camera) is now connected to the $3.6 \mathrm{~m}$ telescope on La Silla via a fibre link and we continue to monitor $\alpha$ Centauri using this instrumental setup. By obtaining more $R V$ measurements over time it will permit us to push these mass limits further down or to detect even a smaller residual $R V$ signal of an orbiting planet.

\section{Summary}

1. We present more than 5 years of high precision differential radial velocities for the two components of the inner binary of the $\alpha$ Centauri system.

2. After subtraction of the binary orbital motion we find the residual $R V$ scatter to be in agreement with our measurement errors. A period search in the $R V$ residuals reveals no significant signals.

3. We set quantitative upper limits for planets around $\alpha$ Centauri A \& B by numerical simulations to establish

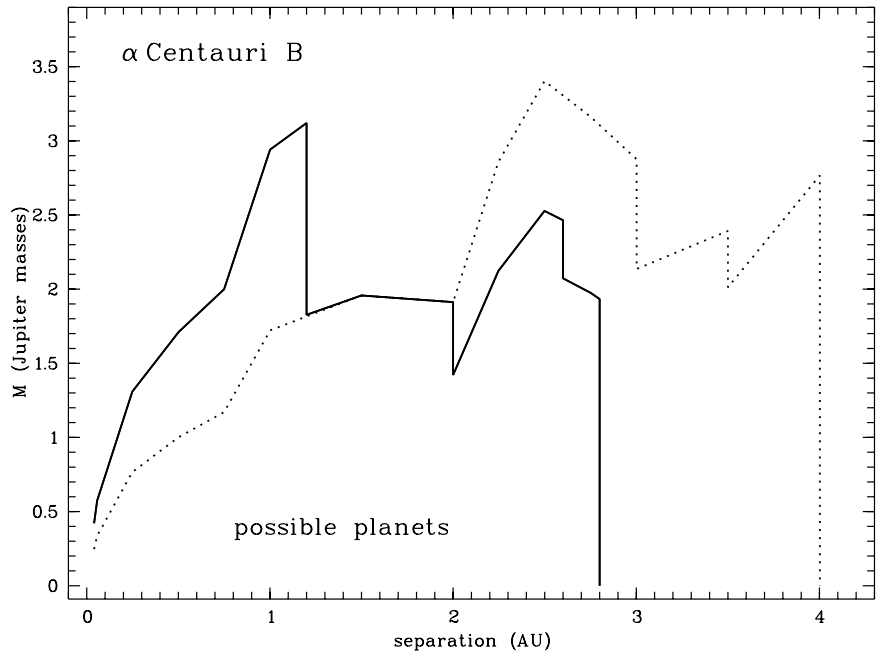

Fig. 8. Zone for possible giant planets orbiting $\alpha$ Centauri B. Again the region defined by the solid line is valid for prograde and the dotted line for retrograde orbits. The presence of planets inside these regions is still possible.

the detection threshold of the CES program. For component A we can exclude planets with $m \sin i>1 M_{\text {Jup }}$ inside $2 \mathrm{AU}$ and planets with $m \sin i>2 M_{\text {Jup }}$ with separations less than $4 \mathrm{AU}$. For component B we rule out the presence of planets having an $m \sin i>$ $1.5 M_{\text {Jup }}$ within $2 \mathrm{AU}$ and up to an orbital radius of $4 \mathrm{AU}$ all planets with $m \sin i>2.5 M_{\text {Jup }}$. In particular we find that "51 Peg-type" planets (i.e. $m \sin i \approx$ $0.5 M_{\text {Jup }}$ at orbital radii less than $0.4 \mathrm{AU}$ ) can be discounted around both stars.

4. In combination with the dynamical limits of Wiegert \& Holman (1997) we exclude all planets with masses larger than $2.5 M_{\text {Jup }}$ around $\alpha$ Centauri $\mathrm{A}$ and all planets with masses larger than $3.5 M_{\text {Jup }}$ around component B. If we assume that possible planets are residing in orbits coplanar with the binary plane, these upper mass limits reach down into the sub-Saturn mass regime at smaller orbital separations.

Acknowledgements. We thank the ESO OPC for generous allocation of observing time to the CES planet search program. We are very grateful to K. Dennerl and S. Döbereiner, who performed part of the observations of the CES program. M. Endl acknowledges support by the Austrian Fond zur Förderung der wissenschaftlichen Forschung N. S7302. Artie P. Hatzes and William D. Cochran acknowledge support from NSF Grant AST-9808980 and NASA Grant NAGW-9227.

\section{References}

Anosova, J., Orlov, V. V., \& Pavlova, N. A. 1994, A\&A, 292, 115

Benest, D. 1988, A\&A, 206, 143

Charbonneau, D., Brown, T. M., Latham, D. W., \& Mayor, M. 2000, ApJ, 529, L49

Cochran, W. D., \& Hatzes, A. P. 1999, in Precise Stellar Radial Velocities, IAU Coll. 170, ASP Conf. Ser., 185, ed. J. B. Hearnshaw \& C. D. Scarfe, 113 
Dvorak, R. 1986, A\&A, 167, 379

Endl, M., Kürster, M., \& Els, S. 2000, A\&A, 362, 585

Endl, M., Kürster, M., Els, S., et al. 2001, in Planetary Systems in the Universe, Proc. IAU Symp. 202, ed. A.J. Penny, P. Artymowicz, A.-M. Lagrange, \& S. S. Russell, in press Guenther, D. B., \& Demarque, P. 2000, ApJ, 531, 503

Guinan, E., \& Messina, S. 1995, IAUC, 6259, 2G

Hale, A. 1994, AJ, 107, 306

Hallam, K. L., Aliner, B., \& Endal, A. S. 1991, ApJ, 372, 610

Hatzes, A. P., Kürster, M., Cochran, W. D., Dennerl, K., \& Döbereiner, S. 1996, J. Geophys. Res. (Planets), 101, 9285

Hatzes, A. P., Cochran, W. D., McArthur, B., et al. 2000, ApJ, $544, \mathrm{~L} 145 \mathrm{H}$

Heintz, W. 1982, Observatory, 102, 42

Holman, M. J., \& Wiegert, P. A. 1999, AJ, 117, 621

Kürster, M., Hatzes, A. P., Cochran, W.D., et al. 1994, The ESO Messenger, 76, 51

Kürster, M., Schmitt, J. H. M. M., Cutispoto, G., \& Dennerl, K. 1997, A\&A, 320, 831
Kürster, M., Hatzes, A. P., Cochran, W. D., et al. 1999, A\&A, 344, L5

Kürster, M., Endl, M., Els, S., et al. 2000, A\&A, 353, L33

Lomb, N. R. 1976, Ap\&SS, 39, 477

Marcy, G. W., Fischer, D. A., \& Butler, R. P. 2001, in Planetary Systems in the Universe, Proc. IAU Symp., 202, ed. A. J. Penny, P. Artymowicz, A.-M. Lagrange, \& S. S. Russell, in press

Marzari, F., \& Scholl, H. 2000, ApJ, 543, 328

Mayor, M., \& Queloz, D. 1995, Nature, 378, 355

Murdoch, K. A., Hearnshaw, J. B., \& Clark, M. 1993, ApJ, 413,349

Pourbaix, D., Neuforge-Verheecke, C., \& Noels, A. 1999, A\&A, 344, 172

Queloz, D., Eggenberger, A., Mayor, M., et al. 2000, A\&A, 359, L153

Scargle, J. D. 1982, ApJ, 263, 835

Wiegert, P. A., \& Holman, M. J. 1997, AJ, 113, 1445 